\title{
Hierarchical Self-Assembly of Achiral Amino Acid Derivatives into Dendritic Chiral Nanotwists
}

\author{
Hai Cao, ${ }^{\dagger}$ Quanzi Yuan, ${ }^{\ddagger}$ Xuefeng Zhu, ${ }^{\dagger}$ Ya-Pu Zhao, ${ }^{* \dagger}$ and Minghua Liu* ${ }^{\dagger}$ \\ ${ }^{\dagger}$ Beijing National Laboratory for Molecular Science (BNLMS), Key Laboratory of Colloid, Interface and Chemical thermodynamics, \\ Institute of Chemistry, Chinese Academy of Sciences, Beijing, 100190, P. R. China \\ ${ }^{\ddagger}$ State Key Laboratory of Nonlinear Mechanics, Institute of Mechanics, Chinese Academy of Sciences, Beijing 100190, P. R. China
}

Supporting Information

ABSTRACT: The organogel formation and self-assembly of a glycine-based achiral molecule were investigated. It has been found that the compound could gel organic solvents either at a lower temperature with lower concentration or at room temperature with higher concentration, which showed different self-assembled nanostructures. At a low temperature of $-15{ }^{\circ} \mathrm{C}$, the compound self-assembled into fibrous structures, whereas it formed distinctive flat microbelts at room temperature. When the organogel with nanofibers formed at $-15{ }^{\circ} \mathrm{C}$ was brought into an ambient condition, chiral twist nanostructures were immediately evolved, which subsequently transferred to a giant

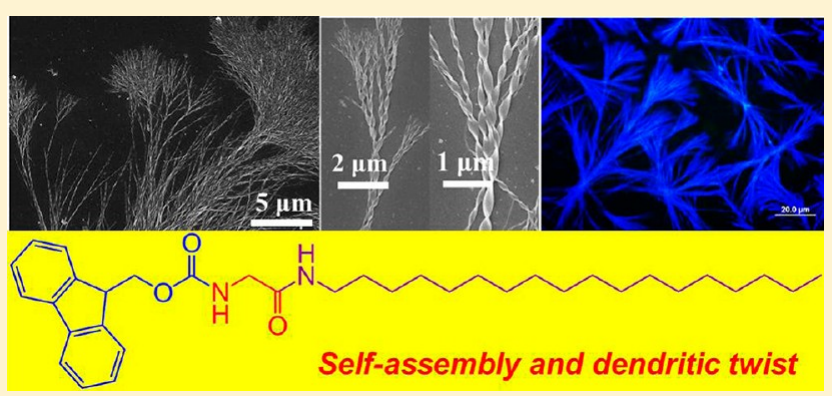
microbelt through a hierarchical dendritic twist with the time. Although the compound is achiral, it formed chiral twist with both left- and right-handed twist structures simultaneously. When a trace analogical chiral trigger, L-alanine or D-alanine derivative, was added, a complete homochiral dendritic twist was obtained. Interestingly, a reverse process, i.e. the transformation of the microbelts into twists, could occur upon dilution of the organogel with microbelt structure. During the dilution, both left- and right-handed chiral twists could be formed again. Interestingly, the same branch from the microbelt formed the twist with the same handedness. A combination of the density functional theory (DFT), molecular mechanics (MM), and molecular dynamics (MD) simulations demonstrates that the temperature-induced twisting of the bilayer is responsible for the morphological transformation and evolution of the dendrite twist. This research sheds new light on the hierarchical transformation of the chiral structures from achiral molecules via controlled self-assembly.

\section{INTRODUCTION}

Helical structures are among the most visually captivating sights found in nature, which can be observed ubiquitously from large celestial structures to sophisticated biological systems. ${ }^{1-8}$ Particularly, the unique structures in biological systems possess specific functions and thus have inspired extensive research interesting in creating artificial mimics. ${ }^{9-12}$ Based on appropriate molecular design and self-assembly processes, many of helical or twist nanostructures have been produced, ${ }^{13-18}$ and various applications of self-assembled helical structures have been developed as a result of their unique properties, such as chiral recognition, ${ }^{19-21}$ asymmetric catalysis, ${ }^{22}$ molecular electronic devices, ${ }^{23-28}$ and template synthesis. ${ }^{29-33}$ In addition, biosystems have frequently switched their structures hierarchically to effectuate different functions. Although significant effort has been devoted to the tuning of the helical structures through external stimuli such as light irradiation, ${ }^{34-36}$ aging, ${ }^{37-39}$ dilution, ${ }^{40}$ or chiral triggering, ${ }^{26,41-45,49}$ it still remains a challenge to hierarchically tune the helical structures through molecular self-assembly.

Helical nanostructures are generally produced by the assembly of intrinsically chiral species or by achiral species with the existence of chiral components. However, some self- assembly processes can cause completely achiral components to form chiral superstructures without any chiral species. In these systems, equal amounts of left-handed (M) and right-handed (P) chiral structures emerge. ${ }^{23,46-52}$ Furthermore, some unusual examples of homochiral structures composed of achiral unit also have been reported recently. However, there still remains mystery, and this provides new opportunities to gain deep insight into the supramolecular chirogenic processes in the self-assembled systems. $53,59,60$

As the only achiral amino acid among all the proteinogenic ones, glycine frequently appears at the termination of $\alpha$-helix as helix break. ${ }^{54}$ So far, hardly any achiral glycine derivatives have been found to form helical structures. In this paper, we designed a long chain glycine derivative with $N$-fluorenyl-9methoxycarbonyl (Fmoc) moiety, abbreviated as FGC18, and investigated their self-assembly in different temperatures and concentration. We selected the glycine to see the unique properties of this amino acids in comparison with our previously designed chiral amino acid derivatives. ${ }^{30,31,56} \mathrm{We}$

Received: August 12, 2012

Revised: October 5, 2012

Published: October 9, 2012 
introduced the Fmoc group to enhance the $\pi-\pi$ interaction during the self-assembly. Thus, the derivative have the $\pi-\pi$ stacking, hydrogen binding, and hydrophobic sites, which should be favorable for the self-assembly. By using a glycinebased achiral molecule, we have successfully achieved the tuning of hierarchical dendritic chiral twist by varying temperature, concentration, and chiral trigger. Figure 1

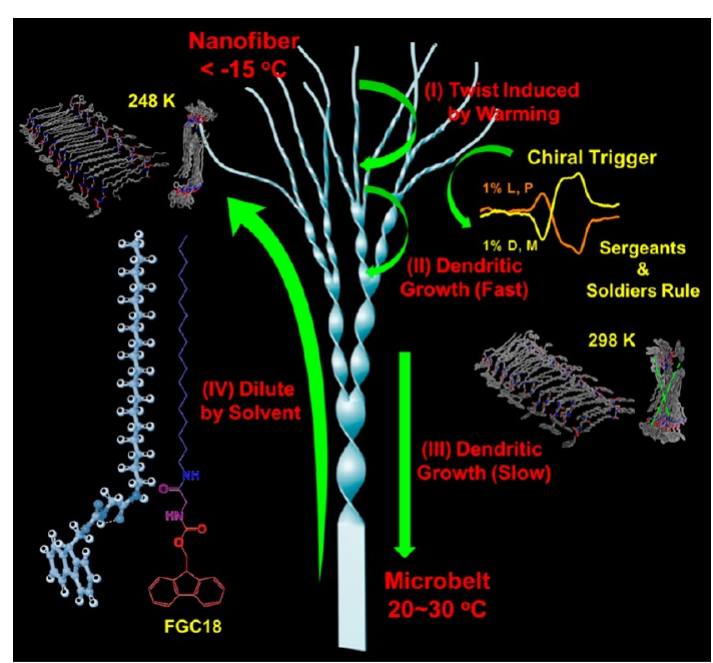

Figure 1. Molecular structure of FGC18 and the schematic illustration of hierarchical dendrite twist structure formed by FGC18. There are two routs of the transformation of the morphologies. When the nanofiber formed at a lower temperature of $-15{ }^{\circ} \mathrm{C}$ was brought to room temperature, it changed into twist (I) and then grew into dendrtici one (II) and larger dendritic twist (III) as well as microbelts. In the second route, the microbelts could be transferred into dendritic twists by dilution. Fast and slow mean the formation speed of the nanostructures.

schematically illustrates such transformations. FGC18 could form organogels in ethyl acetate. The gel could be prepared from a low concentration at low temperature (A, referred to as type A gel hereafter) or a high concentration at room temperature (B, referred to as type B gel hereafter). In type A gel, nanofibers were initially formed, while in type $B$ gel, giant microbelts was formed. When the type A gel was warmed at room temperature, the gel was found to collapse in a few minutes and twist structures formed and grew into dendritic one and finally into microbelts. On the other hand, if initially microbelt was formed as in type B gel, they could be transferred into treelike twist structures upon dilution, depicting a reversible process. So far, the twist formation through the self-assembly has been extensively investigated. Here, we showed the first example of the generation of a reversible hierarchical dendritic twist between a microbelt and twist states, with the control of the chirality.

The experimental characterizations of the gels and the nanostructures using circular dichroism spectra (CD), Fourier transform infrared spectra (FT-IR), and X-ray diffraction (XRD) combined with the density functional theory (DFT), molecular mechanics (MM), and molecular dynamics (MD) simulations were employed to investigate the packing and interaction during the self-assembly process.

\section{RESULTS AND DISCUSSION}

Self-Assembly of FGC18 in Various Organic Solvents. Self-assembly of the glycine-based lipid, FGC18, showed diverse responses to different organic solvents. Precipitate rather than gelation was obtained in various polar solvents including acetonitrile, ethanol, and DMF, with fibrous or amorphous structures (Figure 2A-C). FGC18 was able to

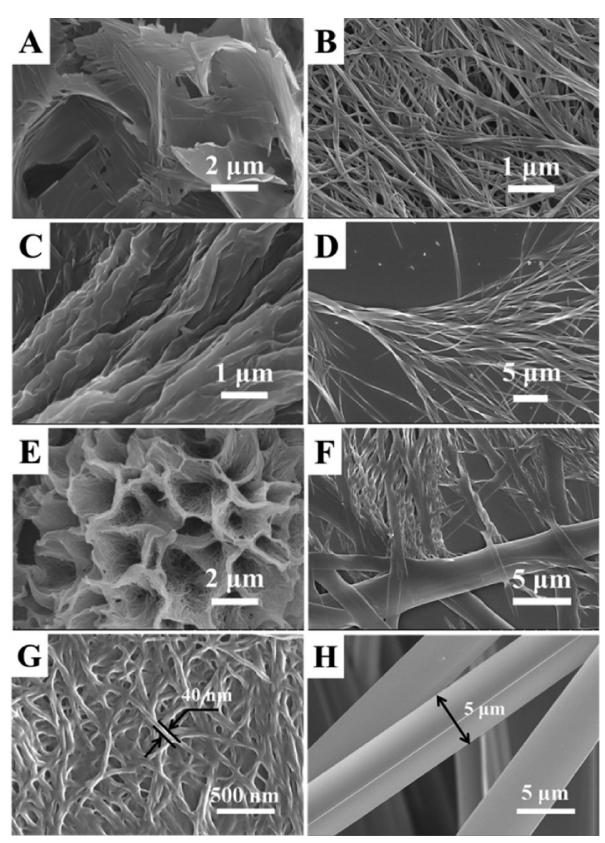

Figure 2. SEM images of nanostructures formed by FGC18 (10 mg/ $\mathrm{mL}$ for $\mathrm{A}-\mathrm{F}, 30 \mathrm{mg} / \mathrm{mL}$ for $\mathrm{G}$ and $\mathrm{H})$. (A-C) Precipitates formed in acetonitrile, ethanol, and DMF, respectively. (D) DMSO gel formed at room temperature. (E, F) The gel and precipitate formed in toluene at $-15{ }^{\circ} \mathrm{C}$ and room temperature in toluene, respectively. (G, H) The xerogel formed in ethyl acetate at low temperature and room temperature, respectively.

gelate some other organic solvents such as DMSO, toluene, and ethyl acetate. Although it lacks a chiral center, FGC18 was able to form twist ribbons in DMSO (Figure 2D), in which both left- and right-handed structures coexisted. When FGC18 formed organogels with toluene (Figure 2E,F) or ethyl ester, the gels showed different behaviors depending on the gel temperature and concentration. The gelation was observed to occur both at lower temperature $\left(-15{ }^{\circ} \mathrm{C}\right)$ with low critical gelation concentration (CGC) and at room temperature with a much higher concentration. This was particularly obvious for ethyl acetate as the solvent, as shown in Figure 2G,H. Specifically, when FGC18 was dissolved in ethyl acetate at a concentration of $5-30 \mathrm{mg} / \mathrm{mL}$ (CGC is about $5 \mathrm{mg} / \mathrm{mL}$ ) through heating and then fast cooling below $-15^{\circ} \mathrm{C}$ within half an hour, a compact gel formed (type A gel). SEM characterization revealed the formation of normal fibrous structures with width of about $40 \mathrm{~nm}$, as shown in Figure 2G. Such features remained constant across varying concentrations (Figure S2). At a higher concentration of $30 \mathrm{mg} / \mathrm{mL}$, however, FGC18 could form a gel at room temperature. Experimentally, the hot FGC18 solution was naturally cooled down to room temperature within 3-4 h, and a white gel was formed (type B gel). In this case, giant microbelts with sizes of a few micrometers in width and hundreds of micrometers in length were obtained (Figure 2H). More interestingly, these morphologies showed a hierarchical transformation upon increasing the temperature, which will be discussed in the following section. 
Dendritic Growth of the Type A Gel at Room Temperature. The type A gel, which formed at a low temperature of $-15{ }^{\circ} \mathrm{C}$ and at a low concentration of $10 \mathrm{mg} /$ $\mathrm{mL}$, showed remarkable changes when exposed to room temperature. When it was taken out and kept naturally at ambient condition, the gel collapsed in a few minutes and then experienced a phase separation, resulting in precipitation. We analyzed the collapsed gel at different time intervals as well as the final precipitate using the SEM, as shown in Figure 3 and

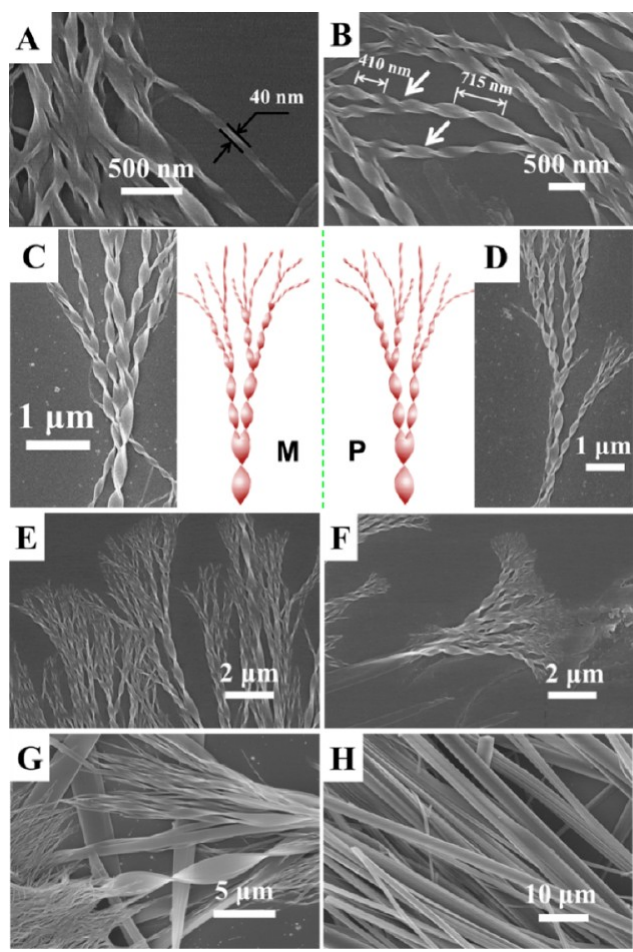

Figure 3. SEM images of the gel $(10 \mathrm{mg} / \mathrm{mL})$ formed at low temperature transferred to ambient circumstance in $(\mathrm{A}-\mathrm{F}) 10 \mathrm{~min}$, (G) $2 \mathrm{~h}$, and (H) 2 days. Dendritic twists with both left-handed (the left side, except $\mathrm{G}$ ) and right-handed chirality were obtained (the right side).

Figures S3 and S4. Immediately after the collapse, the twist structures of widths in the range of $40 \mathrm{~nm}$ to over $1 \mu \mathrm{m}$ were observed, and hardly any nanofibers could be detected. The twist could be singular or entangled with others. The minimum twist structure is about $40 \mathrm{~nm}$ in width, which corresponds to the dimensions of nanofibers, suggesting that twisting of the nanofibers is responsible for the twist formation.
These initial twists tended to grow into higher-order structures or dendritic twists. In $10 \mathrm{~min}$, a clear dendritic twist was formed. When the sample was kept at room temperature for $2 \mathrm{~h}$, phase separation occurred. Further analyzing the upper clear solution revealed the formation of twist structure, as shown in Figure S6A, while the precipitates were composed of the twists with different sizes, as shown in Figure 3G. Some microbelts were also observed simultaneously. After 2 days, precipitates were completely dominated by microbelt structures, as shown in Figure $3 \mathrm{H}$. This indicated that upon aging of the type $\mathrm{A}$ gel at room temperature the initially assembled nanofibers grew gradually into the twist, dendritic twist, and finally microbelts. It is worth noting that the dendritic structures were formed rapidly while the microbelts were formed through days of aging. When the type A gel was aged for several days at room temperature, an equilibrium between the precipitate and the clear solution reached. In this case, casting of the clear solution still appeared as twist structures (Figure S6C,D), while the precipitates were completely composed of the microbelt structure, which was the final structure. In addition, though the type A gel has a large concentration range, similar changes were observed under different concentrations, for example $20 \mathrm{mg} / \mathrm{mL}$ (Figure S6BD).

During the growth from nanofibers to the microbelt, both left- and right-handed twist structures appeared with approximately equal probability as a result of the achiral nature of FGC18. Interestingly, although both left-handed and righthanded twist structures could be observed, all the twist structures in a certain branch had the same chirality. As shown in Figure 3A,C,E, some of the dendritic twists appeared as left-handed ones, while the others as right-handed twists in Figure 3B,D,F. This implies that during the self-assembly the initially formed seed twist was produced by chance, while the growing twists followed the chirality of the initial seeds.

In addition, since the dimensions of the dendritic twists were large enough to be observed by using conventional optical microscopy, we followed these processes under optical microscopy as well, as shown in Figure 4A,B. Moreover, the dendritic structures displayed photoluminescence when excited with UV light, which provided an alternative approach to capture the fluorescence image of these twists. Beautiful dendritic structures could be observed during the warming process, which were consistent with the SEM measurements.

Control of the Chirality by Chiral Seed Dopant. Although we obtained the twist from the achiral FGC18, the chirality of each twist appeared by chance. In order to control the chirality of the twists, we added a chiral analogue, L or D-

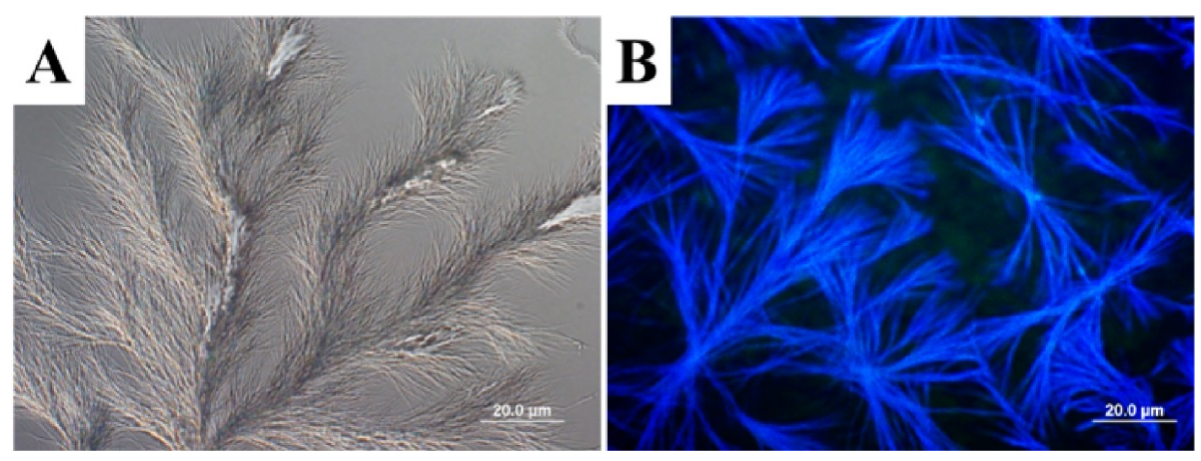

Figure 4. (A) Microscopic and (B) fluorescence microscopic images of the dendritic twists formed during step I and step II. 
FAC18, whose structures are shown in Figure 5, into the system and found that the chirality of each twist was controlled
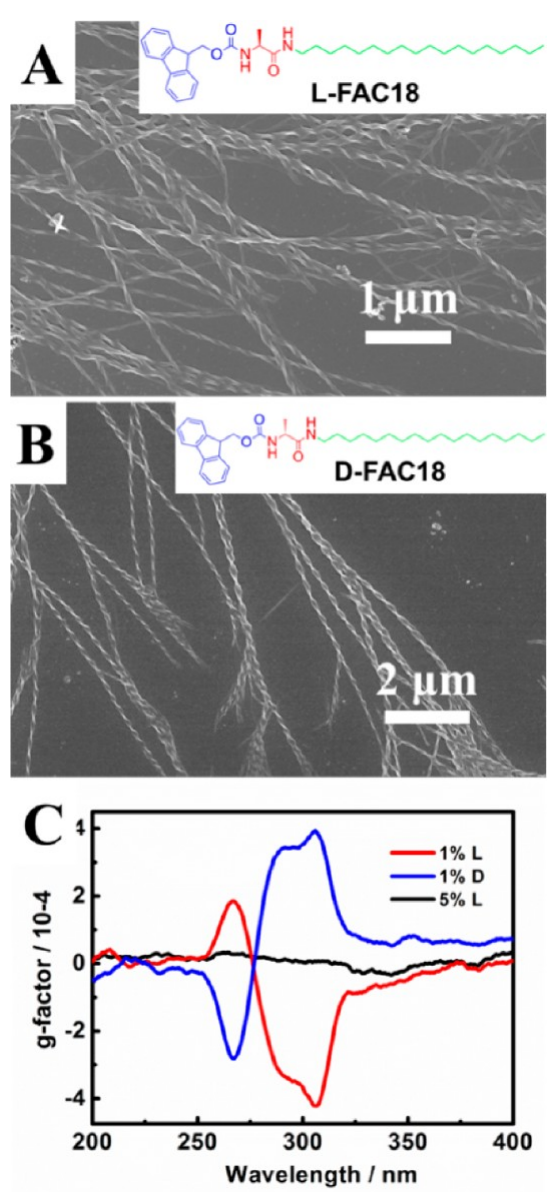

Figure 5. SEM images of the type A gel $(10 \mathrm{mg} / \mathrm{mL})$ doped with (A) $1 \%$ of L-FAC18 and (B) $1 \%$ of D-FAC18 respectively in steps I and II. (C) CD spectra of the collapsed gel doped with various molar ratios of chiral analogues. L-FAC18 and D-AC18 are abbreviated to L and D, respectively.

completely. The doping of 1\% L-FAC18 or D-FAC18 with FGC18 had no effect on the emergence of twist structures but significantly controlled the helicity of the twist, as shown in Figure 5A,B. Only right-handed twist appeared after doping with $1 \%$ L-FAC18, while the opposite left-handed twist came out when doped with D-enantiomer. Such control of the chirality was further confirmed by the macroscopic measurement using CD spectroscopy, as shown in the Figure 5C. An exciton couplet with positive Cotton effect at $267 \mathrm{~nm}$ and two negative ones at 288 and $305 \mathrm{~nm}$, with a crossover at $276 \mathrm{~nm}$ was obtained when we used 1\% L-FAC18 as dopant. A mirrorimaged CD spectrum was obtained when $1 \%$ D-FAC18 was used. Thus, following the sergeant-soldier principle, ${ }^{26,43,48,55}$ we successfully fabricated well-defined homochiral dendritic twist structures.

It should be noted that over doping of the chiral analogue into the system destroyed the twist structure. The CD intensity dramatically decreased and even disappeared after 5\% L-FAC18 was mixed. Subsequently, flat fibrous structures appeared to be dominant, as shown in Figure S14.

Dendritic Twist Formation by Diluting the Microbelts with Ethyl Acetate. As described above, in type B gel, the compound self-assembled into the microbelts, and there existed a balance between the microbelts and the clear solution. Interestingly, when the gel with these microbelts structures was diluted by ethyl acetate followed by gentle shaking, the transformation from microbelts to dendritic twist was observed, as shown in Figure 6. The splitting of the microbelt into the
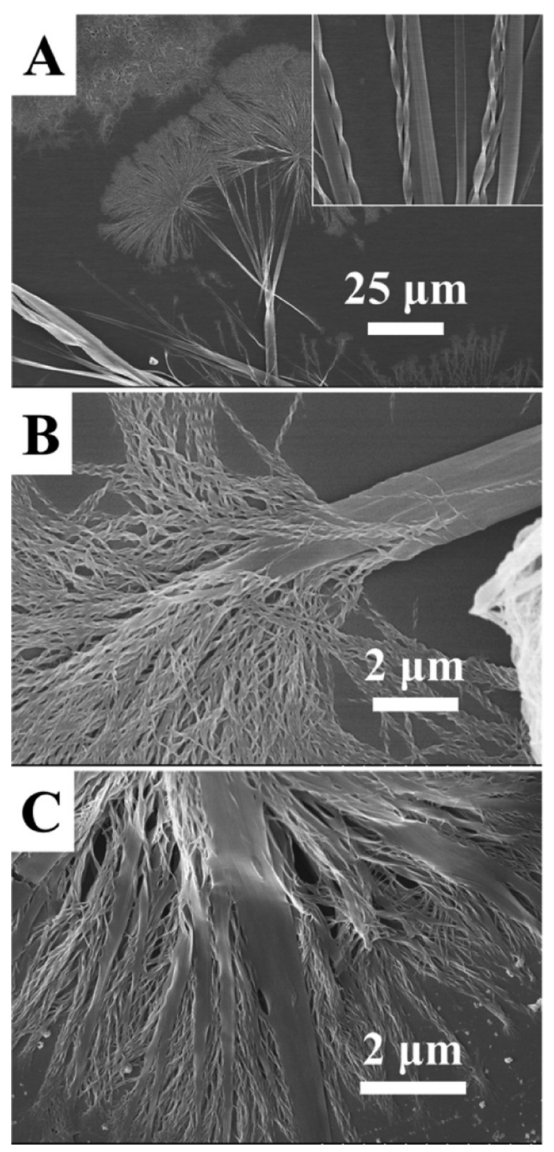

Figure 6. SEM images of the dendritic twists formed by (A, B) mixing and stirring the microbelts with upper liquid or (C) diluting the precipitate by 2 -fold ethyl acetate exposure wherein, the split of the microbelts into twist structures is obvious. Inset: a close-up image of the branch in (A). In all these cases, the microbelts were obtained by cooling the FGC18 solution with concentration of $20 \mathrm{mg} / \mathrm{mL}$ at room temperature for more than $2 \mathrm{~h}$. All the twists in (A) and (C) are righthanded, while left-handed ones are present in (B).

dendritic twists was ubiquitous. Similarly, both left- and righthanded twists were formed. However, all the twists that split from the same microbelt had the same helicity. Furthermore, when the type B gel was heated gently, it induced the formation of dendritic twists. These results clearly indicate that the twists and the microbelts could be reversibly transformed.

FT-IR and X-ray Diffraction Data of the Nano/Micro Structures. To gain further insights into the structural transformation, FT-IR and XRD measurements were performed. Since the nanofiber, twist, and microbelts patterns could be obtained in a controlled manner, we selected these three structures and measured their FT-IR and XRD. The FTIR spectra illustrated clear changes in hydrogen bonds during the morphological transformation, as shown in Figure 7A. The $\mathrm{N}-\mathrm{H}$ stretching band was found at 3307,3311 , and $3322 \mathrm{~cm}^{-1}$ for the nanofiber, microbelt, and nanotwist, respectively. This implies the sequentially decreasing $\mathrm{H}$-bond length in the order of nanofiber, microbelt, and twist. Similar changes were found 

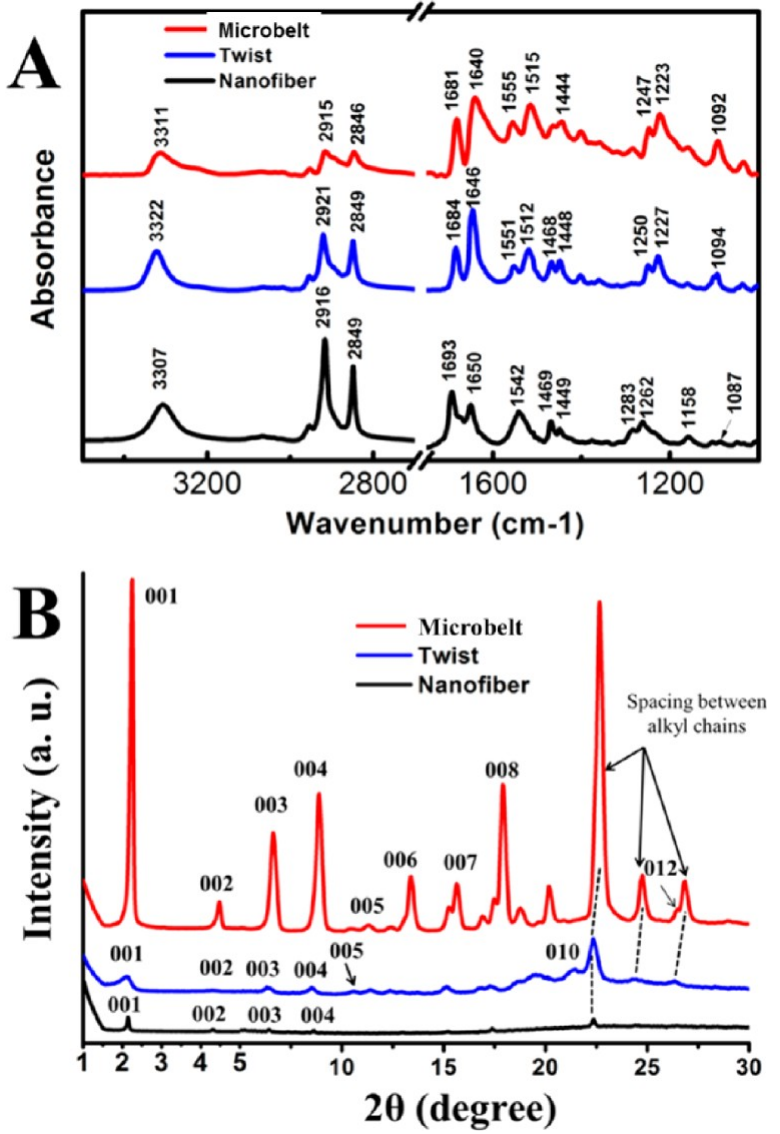

Figure 7. (A) Infrared spectra and (B) XRD patterns of nanofibers, twists, and microbelts.

in the asymmetrical and symmetrical $\mathrm{CH}_{2}$ stretching band of the alkyl group, suggesting that the alkyl chain became disordered after twist formation, which were however orderly packed after the twists turn into microbelts. Other apparent changes were the shift and relative intensities of two $\mathrm{C}=\mathrm{O}$ stretching bands, which appeared at 1693 and $1649 \mathrm{~cm}^{-1}$ for the nanofibers, while these vibrational bands shifted to 1683 and $1645 \mathrm{~cm}^{-1}$ for the twists, suggesting that the $\mathrm{C}=\mathrm{O}$ groups of the FMOC group are mainly responsible for the formation of hydrogen bonds within the nanofibers while the hydrogen bonds between amide groups play a key role in the twist formation. Interestingly, all these hydrogen bonds were enhanced, and orderly $\beta$-sheet secondary structure formed upon the formation of microbelts.

The X-ray diffraction shown in Figure 7B revealed welldefined diffraction patterns for all three structures, which is typical of layered structures. Based on Bragg's equation, the layer distance or $d$ spacing was estimated to be $4.12 \mathrm{~nm}$ for the nanofibers, indicating the formation of interdigitated bilayer structure. ${ }^{56}$ The $d$ spacing changed into 4.16 and $3.92 \mathrm{~nm}$ for the twists and the microbelts, respectively, suggesting that the bilayer structure was perturbed. The broad peak appeared for the twists might due to their discrete sizes. The peak with $2 \theta=$ $22.36(0.40 \mathrm{~nm})$ for the nanofibers is assigned to the spacing between the alkyl chains. Accordingly, three reflections were detected in the $2 \theta$ range of $22^{\circ}-27^{\circ}$ for the twists and microbelts. ${ }^{57}$ The changes of these reflections indicate that the spacing between alkyl chains are slightly enlarged to produce the dendritic twist and then compressed to form microbelts, which is in in accordance to the infrared spectra. It should be noted that the microbelts showed remarkable intense XRD signals as a result of their orderly and compact arrangement.

Based on the spectral data, the morphology transformation mechanism can be illustrated as follows: when hot ethyl acetate solution of FGC18 was fast cooled to low temperature, nanofibers with the strongest intermolecular hydrogen bonding were formed as a result of the thermodynamic driving assembly. The molecular packing and the hydrogen bonding mode changes as temperature rise, resulting in the formation of twist structures. The hydrogen bonding was enhanced as time passed by and finally gave birth to the nanobelts, which are the most regular and stable structures.

Molecular Modeling of Twist Formation. In order to get a better understanding of the mechanisms of the molecular packing and twist formation in FGC18 stacks from the atomic level, a combination method of the density functional theory (DFT), molecular mechanics (MM), and molecular dynamics (MD) simulations was employed. The optimized configuration of FGC18 was obtained using DFT calculations, as shown in Figure S11. To obtain the most stable topology of the FGC18 stacks, a variety of initial configurations of the FGC18 stacks (32 of the optimized FGC18s, $\sim 8 \mathrm{~nm}$ in length, and $\sim 4.5 \mathrm{~nm}$ in width) were relaxed using $\mathrm{MM}$ simulations to minimize the energy at $0 \mathrm{~K}$. The initial FGC18 stacks were parallel to each other, as shown in Figure 8A.
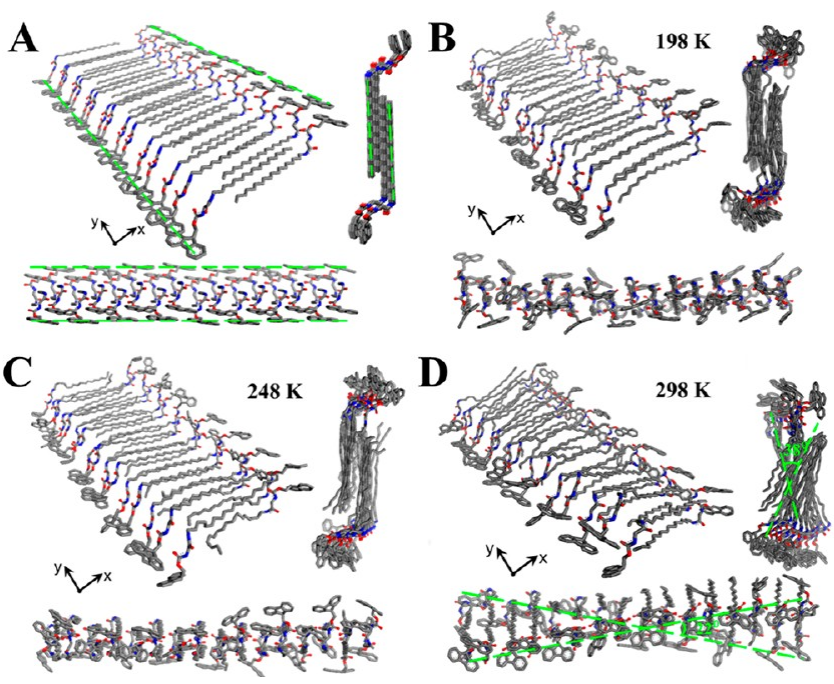

Figure 8. Total view, side view in $y$ direction, and side view in $x$ direction of (A) the initial configuration of FGC18 stacks, (B) the relaxed configuration of the FGC18 stacks at $T=198 \mathrm{~K}$, (C) $T=248$ $\mathrm{K}$, and (D) $T=298 \mathrm{~K}$. The configurations are displayed in stick style. The gray, red, and blue sticks represent the carbon, oxygen, and nitrogen atoms, respectively. The hydrogen atoms are not shown for the sake of convenience. The green lines represent the directions of the FGC18s.

Based on the most stable initial configuration, MD simulations were carried out at low temperatures of $198 \mathrm{~K}$, $248 \mathrm{~K}$, and a high temperature of $298 \mathrm{~K}$ (Figure 8B-D). The canonical ensemble (NVT ensemble: constant number of atoms, volume, and temperature) was used. The Andersen thermostat with a time-step of $1 \mathrm{fs}$ was employed to regulate the temperature. All simulations were performed for $1 \mathrm{~ns}$ to get a fully relaxed configuration using PCFF (the polymer consistent force field), ${ }^{58}$ whose parameters are mostly derived 
from ab initio data using a least-squares fitting technique and are intended for polymers and organic materials applications.

As shown in Figure 8, the FGC18 stacks remained parallel to each other and exhibited an achiral topology at low temperature ( $T=198$ and $248 \mathrm{~K}$ ), although the FGC18 stacks vibrated due to the temperature fluctuation. The FGC18 was stable at low temperatures, which is consistent with the appearance of the smooth fibrous structures observed in experiments. When the temperature was increased, a dramatic transformation took place. The originally parallel array of the alkyl chains was twisted, and the FGC18 stack transformed into a helical structure at room temperature $(T=298 \mathrm{~K})$. The angle between the two ends of the stack was about $38^{\circ}$ for the total length of about $8 \mathrm{~nm}$, which implied that the period of the simulated chiral structure was about $76 \mathrm{~nm}$.

Then, in order to gain further insight into the handedness and evolution of the twist during the self-assembly process, two of the FGC18 stacks were put together to build up a larger stack at a high level and relaxed in the MD simulations for $1 \mathrm{~ns}$ at room temperature $(T=298 \mathrm{~K})$, as shown in Figure 9. Two
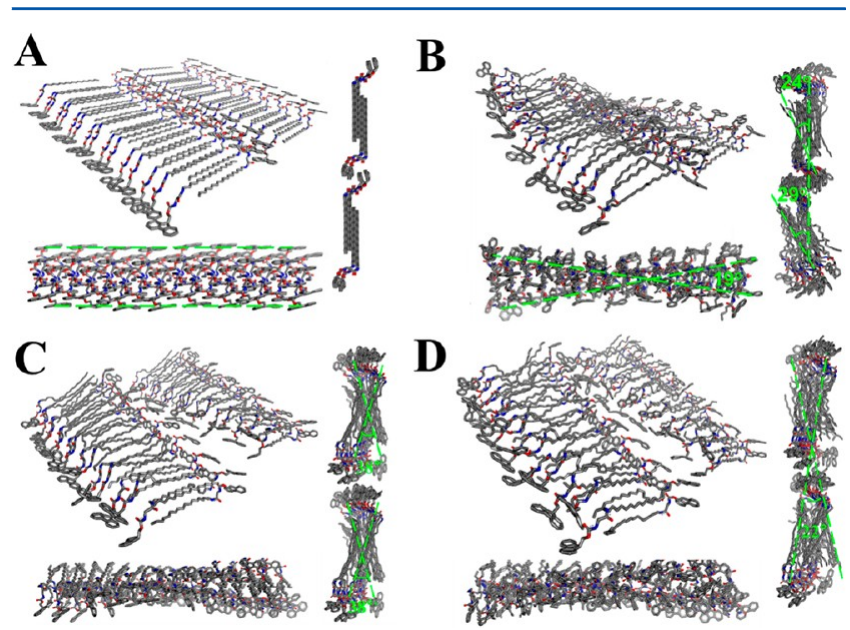

Figure 9. (A, B) Initial configuration and relaxed configuration of double FGC18 bilayer stacks at $T=298 \mathrm{~K}$. The angles $24^{\circ}$ and $29^{\circ}$ represent the twist angle of alkyl chains of the two bilayer stacks individually, while $19^{\circ}$ represents the twist angle of the fluorene groups. (C, D) Initial configuration and relaxed configuration of double twist FGC18 bilayer stacks with both twist angles of $38^{\circ}$ at $T=$ $298 \mathrm{~K}$. After relaxed in the MD simulations for $1 \mathrm{~ns}$, smaller twist angle of $22^{\circ}$ compared to an individual bilayer stacking was obtained.

of the initial FGC18 stacks were placed side by side and relaxed at room temperature, as shown in Figure 9A,B. The fluorene groups of neighboring FGC18 stacks interacted with each other, making the two FGC18 stacks twist from flat to form a helical structure. A bilayer at neighborhood was found to have a significant effect on the twist angle of the other one and vice versa. The same handedness adopt by adjacent bilayer might be responsible for the twist formation and also the homohanded twist appeared at restricted area. The twist angles of a single bilayer and a combination of such bilayers were about $24^{\circ}$ and $29^{\circ}$, respectively, smaller than that of an independent FGC18 bilayer stack. The period of the simulated chiral structure was about $100 \mathrm{~nm}$. Since the sizes of the twists were much more than two adjacent bilayers, the final pitch observed by SEM was much larger as a result of multiple bilayer interactions.

Since the merging or splitting of the twist was always accompanied by variation of the pitch, two preoptimized twist bilayers packed side by side were thus selected to simulate the dendritic growth of hierarchical structures (Figure 9C,D). Though the helicity remained unchanged, the twisting angle relaxed significantly after $1 \mathrm{~ns}$ of dynamics. The twist angle of the combination was $22^{\circ}$, about half of that of the initial single bilayer, which implies that the pitch was doubled after the evolution. These are consistent with the experimental observation that the pitch was doubled after two twist entangled with others to produce a larger one. The MD simulations clearly showed that the interactions of the fluorene groups between adjacent bilayers gave rise to the variation of pitch during dendritic evolution. Therefore, the twisting of bilayer is responsible for the emergence of twist nanostructures while the fluorenyl groups of neighboring FGC18 bilayer, which stack and interact with each other, play a key role for the dendritic growth. After subsequent multiple growth processes, the twisted angle became very small while the pitch turned very large, and finally the microbelts formed as a thermodynamically stable structure.

It should be noted that similar morphologies appeared in DMSO and toluene, so solvent effect was not took into consideration in all the above cases. However, satisfied results were obtained.

\section{CONCLUSIONS}

In conclusion, a simple achiral glycine derivative is designed and found to self-assemble into nanofibers at a lower concentration and lower temperature and giant microbelts at a higher concentration, respectively. More interestingly, the nanofiber could grow into one-dimensional dendritic nanotwist, while microbelt could also be diluted into the nanotwists. During the formation of the dendritic twist, both $\mathrm{P}$ and $\mathrm{M}$ helicity formed simultaneously. Doping of trace chiral trigger could induce the emergence of homochiral dendritic twist according to the sergeants and soldiers principle. During the change of the microbelt into the dendritic twist, the twist with the same chirality was obtained from the same microbelt. Density functional theory (DFT), molecular mechanics (MM), and molecular dynamics (MD) simulations demonstrated that the temperature-induced twisting of the bilayer was responsible for the morphological transformation and dendritic growth of the dendritic twist. Present results on the evolution of hierarchically self-assembled dendritic nanostructures can deepen the understanding of controllable and hierarchical self-assembly process.

\section{ASSOCIATED CONTENT}

\section{S Supporting Information}

Synthesis, characterization, and complementary SEM images. This material is available free of charge via the Internet at http://pubs.acs.org.

\section{AUTHOR INFORMATION}

\section{Corresponding Author}

*E-mail liumh@iccas.ac.cn (M.L.), yzhao@imech.ac.cn (Y.P.Z.).

\section{Notes}

The authors declare no competing financial interest.

\section{ACKNOWLEDGMENTS}

This work was supported by National Basic Research Program (No. 2007CB808005, 2006CB932101), the National Natural 
Science Foundation of China (Nos. 20533050, 20773141, 11072244), and the Fund of the Chinese Academy of Sciences.

\section{REFERENCES}

(1) Snir, Y.; Kamien, R. D. Entropically Driven Helix Formation. Science 2005, 307, 1067-1067.

(2) Smith, D. K. Lost in translation? Chirality Effects in the SelfAssembly of Nanostructured Gel-Phase Materials. Chem. Soc. Rev. 2009, 38, 684-694.

(3) Lee, C. C.; Grenier, C.; Meijer, E. W.; Schenning, A. P. H. J. Preparation and Characterization of Helical Self-Assembled Nanofibers. Chem. Soc. Rev. 2009, 38, 671-683.

(4) Yang, M.; Kotov, N. A. Nanoscale Helices from Inorganic Materials. J. Mater. Chem. 2011, 21, 6775-6792.

(5) Ariga, K.; Hill, J. P.; Lee, M. V.; Vinu, A.; Charvet, R.; Acharya, S. Challenges and Breakthroughs in Recent Research on Self-Assembly. Sci. Technol. Adv. Mater. 2008, 9, 014109.

(6) Ariga, K.; Michinobu, T.; Nakanishi, T.; Hill, J. P. Chiral Recognition at the Air-Water Interface. Curr. Opin. Colloid Interface Sci. 2008, 13, 23.

(7) Kuhn, H. Origin of life - Symmetry Breaking in the Universe: Emergence of Homochirality. Curr. Opin. Colloid Interface Sci. 2008, 13, 3-11.

(8) Praveen, V. K.; Babu, S. S.; Vijayakumar, C.; Varghese, R.; Ajayaghosh, A. Helical Supramolecular Architectures of SelfAssembled Linear $\pi$-Systems. Bull. Chem. Soc. Jpn. 2008, 81, 11961211

(9) Engelkamp, H.; Middelbeek, S.; Nolte, R. J. M. Self-Assembly of Disk-Shaped Molecules to Coiled-Coil Aggregates with Tunable Helicity. Science 1999, 284, 785-788.

(10) Cornelissen, J. J. L. M.; Rowan, A. E.; Nolte, R. J. M.; Sommerdijk, N. A. J. M. Chiral Architectures from Macromolecular Building Blocks. Chem. Rev. 2001, 101, 4039-4070.

(11) Amabilino, D. B. Chirality at the Nanoscale; Wiley-VCH: Weinheim, 2009.

(12) Crego-Calama, M.; Reinhoudt, D. N. Supramolecular Chirality. Topics in Current Chemistry; Springer: Berlin, 2006.

(13) Lee, E. J.; Hammer, B.; Kim, J. K.; Page, Z.; Emrick, T.; Hayward., R. C. Hierarchical Helical Assembly of Conjugated Poly(3hexylthiophene)-block-poly(3-triethylene glycol thiophene) Diblock Copolymers. J. Am. Chem. Soc. 2011, 133, 10390-10393.

(14) Danila, I.; Riobé, F.; Piron, F.; Josep Puigmartí-Luis, J.; Wallis, J. D.; Linares, M.; Ågren, H.; Beljonne, D.; Amabilino, D. B.; Avarvari, N. Hierarchical Chiral Expression from the Nano- to Mesoscale in Synthetic Supramolecular Helical Fibers of a Nonamphiphilic $\mathrm{C}_{3^{-}}$ Symmetrical $\pi$-Functional Molecule. J. Am. Chem. Soc. 2011, 133, 8344-8353.

(15) Li, C.; Deng, K.; Tang, Z. Y.; Jiang, L. Twisted Metal-Amino Acid Nanobelts: Chirality Transcription from Molecules to Frameworks. J. Am. Chem. Soc. 2010, 132, 8202-8209.

(16) Wang, J.; Lu, H.; Kamat, R.; Pingali, S. V.; Urban, V. S.; Cheng, J. J.; Lin, Y. Supramolecular Polymerization from Polypeptide-Grafted Comb Polymers. J. Am. Chem. Soc. 2011, 133, 12906-12909.

(17) George, S. J.; Ajayaghosh, A.; Jonkheijm, P.; Schenning, A. P. H. J.; Meijer, E. W. Coiled-Coil Gel Nanostructures of Oligo-(pphenylenevinylene)s: Gelation-Induced Helix Transition in aHigherOrder Supramolecular Self-Assembly of a Rigid $\pi$-Conjugated System. Angew. Chem., Int. Ed. 2004, 43, 3422-3425.

(18) Ajayaghosh, A.; Chithra, P.; Varghese, R. Self-Assembly of Tripodal Squaraines: Cation-Assisted Expression of Molecular Chirality and Change from Spherical to Helical Morphology. Angew. Chem., Int. Ed. 2007, 46, 230-233.

(19) Yashima, E.; Maeda, K.; Iida, H.; Furusho, Y.; Nagai, K. Helical Polymers: Synthesis, Structures, and Functions. Chem. Rev. 2009, 109, 6102-6211.

(20) Yashima, E.; Maeda, K.; Furusho, Y. Single- and DoubleStranded Helical Polymers: Synthesis, Structures, and Functions. Acc. Chem. Res. 2008, 41, 1166-1180.
(21) Fenniri, H.; Deng, B. L.; Ribbe, A. E. Helical Rosette Nanotubes with Tunable Chiroptical Properties. J. Am. Chem. Soc. 2002, 124, 11064-11072.

(22) Sato, I.; Kadowaki, K.; Urabe, H.; Jung, J. H.; Ono, Y.; Shinkai, S.; Soaia, K. Highly Enantioselective Synthesis of Organic Compound Using Right- and Left-Handed Helical Silica. Tetrahedron Lett. 2003, $44,721-724$.

(23) Di Maria, F.; Olivelli, P.; Gazzano, M.; Zanelli, A.; Biasiucci, M.; Gigli, G.; Gentili, D.; D’Angelo, P.; Cavallini, M.; Barbarella, G. A Successful Chemical Strategy to Induce Oligothiophene Self-Assembly into Fibers with Tunable Shape and Function. J. Am. Chem. Soc. 2011, $133,8654-8661$.

(24) Ozawa, H.; Tanaka, H.; Kawao, M.; Unoa, S.; Nakazato, K. Preparation of Organic Nanoscrews from Simple Porphyrin Derivatives. Chem. Commun. 2009, 7411-7413.

(25) Li, X. Q.; Stepanenko, V.; Chen, Z. J.; Prins, P.; Siebbelesb, L. D. A.; Würthner, F. Functional Organogels from Highly Efficient Organogelator Based on Perylene Bisimide Semiconductor. Chem. Commun. 2006, 3871-3873.

(26) Yamamoto, T.; Fukushima, T.; Kosaka, A.; Jin, W.; Yamamoto, Y.; Ishii, N.; Aida, T. Conductive One-Handed Nanocoils by Coassembly of Hexabenzocoronenes: Control of Morphology and Helical Chirality. Angew. Chem., Int. Ed. 2008, 47, 1672-1675.

(27) Schenning, A. P. H. J.; Meijer, E. W. Supramolecular Electronics; Nanowires from Self-Assembled $\pi$-conjugated systems. Chem. Commun. 2005, 3245-3258.

(28) Babu, S. S.; Prasanthkumar, S.; Ajayaghosh, A. Self-Assembled Gelators for Organic Electronics. Angew. Chem., Int. Ed. 2012, 51, 1766.

(29) Jung, J. H.; Parka, M.; Shinkai, S. Fabrication of Silica Nanotubes by Using Self-Assembled Gels and Their Applications in Environmental and Biological Fields. Chem. Soc. Rev. 2010, 39, 42864302.

(30) Gao, P.; Zhan, C. L.; Liu, M. H. Controlled Synthesis of Double- and Multiwall Silver Nanotubes with Template Organogel from a Bolaamphiphile. Langmuir 2006, 22, 775-779.

(31) Jiang, J.; Wang, T. Y.; Liu, M. H. Creating Chirality in the Inner Walls of Silica Nanotubes through a Hydrogel Template: Chiral Transcription and Chiroptical Switch. Chem. Commun. 2010, 46, $7178-7180$

(32) Banerjee, S.; Das, R. K.; Maitra, U. Supramolecular Gels in action'. J. Mater. Chem. 2009, 19, 6649-6687.

(33) Llusar, M.; Sanchez, C. Inorganic and Hybrid Nanofibrous Materials Templated with Organogelators. Chem. Mater. 2008, 20, $782-820$.

(34) Li, L. S.; Jiang, H. Z.; Messmore, B. W.; Bull, S. R.; Stupp, S. I. A Torsional Strain Mechanism To Tune Pitch in Supramolecular Helices. Angew. Chem., Int. Ed. 2007, 46, 5873-5876.

(35) Muraoka, T.; Cui, H. G.; Stupp, S. I. Quadruple Helix Formation of a Photoresponsive Peptide Amphiphile and Its LightTriggered Dissociation into Single Fibers. J. Am. Chem. Soc. 2008, 130, 2946-2947.

(36) Iwaura, R.; Shimizu, T. Reversible Photochemical Conversion of Helicity in Self-Assembled Nanofibers from a 1, w-Thymidylic Acid Appended Bolaamphiphile. Angew. Chem., Int. Ed. 2006, 45, 46014604.

(37) Pashuck, E. T.; Stupp, S. I. Direct Observation of Morphological Tranformation from Twisted Ribbons into Helical Ribbons. J. Am. Chem. Soc. 2010, 132, 8819-8821.

(38) Ziserman, L.; Lee, H. Y.; Raghavan, S. R.; Mor, A.; Danino, D. Unraveling the Mechanism of Nanotube Formation by Chiral SelfAssembly of Amphiphiles. J. Am. Chem. Soc. 2011, 133, 2511-2517.

(39) Adamcik, J.; Castelletto, V.; Bolisetty, S.; Hamley, I. W.; Mezzenga, R. Direct Observation of Time-Resolved Polymorphic States in the Self-Assembly of End-Capped Heptapeptides. Angew. Chem., Int. Ed. 2011, 50, 5495-5498.

(40) Cui, H. G.; Muraoka, T.; Cheetham, A. G.; Stupp, S. I. SelfAssembly of Giant Peptide Nanobelts. Nano Lett. 2009, 9, 945-951. 
(41) Ajayaghosh, A.; Varghese, R.; Mahesh, S.; Praveen, V. K. From Vesicles to Helical Nanotubes: A Sergeantand-Soldiers Effect in the Self-Assembly of Oligo(p-phenyleneethynylene)s. Angew. Chem., Int. Ed. 2006, 45, 7729-7732.

(42) Yan, Y.; Deng, K.; Yu, Z.; Wei, Z. X. Tuning the Supramolecular Chirality of Polyaniline by Methyl Substitution. Angew. Chem., Int. Ed. 2009, 48, 2003-2006.

(43) Palmans, A. R. A.; Meijer, E. W. Amplification of Chirality in Dynamic Supramolecular Aggregates. Angew. Chem., Int. Ed. 2007, 46, 8948-8968.

(44) Ajayaghosh, A.; Reji Varghese, R.; George, S. J.; Vijayakumar, C. Transcription and Amplification of Molecular Chirality to Oppositely Biased Supramolecular $\pi$ Helices. Angew. Chem., Int. Ed. 2006, 45, $1141-1144$

(45) Oda, R.; Huc, I.; Schmutz, M.; Candau, S. J.; MacKintosh, F. C. Tuning Bilayer Twist Using Chiral Counterions. Nature 1999, 399, 566-569.

(46) Murnen, H, K.; Rosales, A. M.; Jaworski, J. N.; Rachel A. Segalman, R. A.; Zuckermann, R. N. Hierarchical Self-Assembly of a Biomimetic Diblock Copolypeptoid into Homochiral Superhelices. J. Am. Chem. Soc. 2010, 132, 16112-16119.

(47) Yang, W. S.; Chai, X. D.; Chi, L. F.; Liu, X. D.; Cao, Y. W.; Lu, R.; Jiang, Y. S.; Tang, X. Y.; Fuchs, H.; Li, T. J. From Achiral Molecular Components to Chiral Supermolecules and Supercoil Self-Assembly. Chem.-Eur. J. 1999, 5, 1144-1149.

(48) Nam, S. R.; Lee, H. Y.; Hong, J. I. Control of Macroscopic Helicity by Usingthe Sergeants-and-Soldiers Principle in Organogels. Chem.-Eur. J. 2008, 14, 6040-6043.

(49) Kimura, M.; Hatanaka, T.; Nomoto, H.; Takizawa, J.; Fukawa, T.; Tatewaki, Y.; Shirai, H. Self-Assembled Helical Nanofibers Made of Achiral Molecular Disks Having Molecular Adapter. Chem. Mater. 2010, 22, 5732-5738.

(50) Chen, H. B.; Zhou, Y.; Yin, J.; Yan, J.; Ma, Y. G.; Wang, L.; Cao, Y.; Wang, J.; Pei, J. Single Organic Microtwist with Tunable Pitch. Langmuir 2009, 25, 5459-5462.

(51) Srivastava, S.; Santos, A.; Critchley, K.; Kim, K. S.; Podsiadlo, P.; Sun, K.; Lee, J.; Xu, C. L.; Lilly, G. D.; Glotzer, S. C.; Kotov, N. A. Light-Controlled Self-Assembly of Semiconductor Nanoparticles into Twisted Ribbons. Science 2010, 327, 1355-1359.

(52) Hough, L. E.; Jung, H. T.; Krüerke, D.; Heberling, M. S.; Nakata, M.; Jones, C. D.; Chen, D.; Link, D. R.; Zasadzinski, J.; Heppke, G.; Rabe, J. P.; Stocker, W.; Körblova, E.; Walba, D. M.; Glaser, M. A.; Clark, N. A. Helical Nanofilament Phases. Science 2009, 325, 456-460.

(53) Pijper, D.; Feringa, B. L. Control of Dynamic Helicity at the Macro- and Supramolecular Level. Soft Matter 2008, 4, 1349-1372.

(54) Aurora, R.; Srinivasan, R.; Rose, G. D. Rules for $\alpha$-Helix Termination by Glycine. Science 1994, 264, 1126-1130.

(55) Green, M. M.; Peterson, N. C.; Sato, T.; Teramoto, A.; Cook, R.; Lifson, S. A Helical Polymer with a Cooperative Response to Chiral Information. Science 1995, 268, 1860-1866.

(56) Duan, P. F.; Qin, L.; Zhu, X. F.; Liu, M. H. Hierarchical SelfAssembly of Amphiphilic Peptide Dendrons: Evolution of Diverse Chiral Nanostructures Through Hydrogel Formation Over a Wide $\mathrm{pH}$ Range. Chem.-Eur. J. 2011, 17, 6389-6395.

(57) Kameta, N.; Masuda, M.; Minamikawa, H.; Shimizu, T. SelfAssembly and Thermal Phase Transition Behavior of Unsymmetrical Bolaamphiphiles Having Glucose- and Amino-Hydrophilic Headgroups. Langmuir 2007, 23, 4634-4641.

(58) Sun, H.; Mumby, S. J.; Maple, J. R; Hagler, A. T. Dioxygen Activation in Methane Monooxygenase: A Theoretical Study. J. Am. Chem. Soc. 2004, 116, 2978-2987.

(59) Liu, Y. L.; Tang, Z. Y. Nanoscale Biocoordination Polymers: Novel Materials from an Old Topic. Chem.-Eur. J. 2012, 18, 10301037.

(60) Liu, Y.; Ma, W. S.; Liu, W. W.; Li, C.; Liu, Y. L.; Jiang, X. Y.; Tang, Z. Y. Silver(I)-Glutathione Biocoordination Polymer Hydrogel: Effectibe Antibacterial Activity and Improved Cytocompatibility. J. Mater. Chem. 2011, 21, 19214-19218. 